\title{
Persistent Graphs and Consensus Convergence
}

\author{
Guodong Shi and Karl Henrik Johansson
}

\begin{abstract}
This paper investigates the role persistent arcs play for averaging algorithms to reach a global consensus under discrete-time or continuous-time dynamics. Each (directed) arc in the underlying communication graph is assumed to be associated with a time-dependent weight function. An arc is said to be persistent if its weight function has infinite $\mathscr{L}_{1}$ or $\ell_{1}$ norm for continuous-time or discrete-time models, respectively. The graph that consists of all persistent arcs is called the persistent graph of the underlying network. Three necessary and sufficient conditions on agreement or $\epsilon$-agreement are established, by which we prove that the persistent graph fully determines the convergence to a consensus. It is also shown how the convergence rates explicitly depend on the diameter of the persistent graph.
\end{abstract}

Keywords: Consensus, Persistent Graphs, Averaging Algorithms

\section{INTRODUCTION}

Recent years have witnessed wide research interest in the the study averaging algorithms throughout social science [10], [13], [11], [12], computer science [15], [36], [37] and engineering [38], [39], [32], [16], [22], [20], [21]. Agreement seeking has been extensively studied in the literature for both discrete-time and continuous-time models [16], [15], [40], [20], [18], [27], [28], [19], [17], [25], [30], [26].

The communication graph plays an important role in the study of consensus. In most existing work, the arc weights, which reflect the strength of the influence from one node to another, are assumed to either be constant whenever two nodes meet with each other [10], [18], [17], or in a compact set with positive lower and upper bounds [40], [16], [27], [28], [13]. However, in reality, the arc weights may vary in a wide range, and may even fade away since arcs may have different persistency properties. Links can be impulsive, vanishing, persistent, etc. Then an interesting question arises: are there certain arcs which are the ones that actually generate the convergence to consensus and how do their properties influence the convergence rate?

The central aim of the paper is to build a model to classify the arcs in the underlying communication graph, and then give a precise description on how the persistent arcs indeed determine the agreement seeking. We define the persistent graph as the graph having links whose weight functions have infinite $\mathscr{L}_{1}$ or $\ell_{1}$ norm for continuous-time and discrete-time algorithms, respectively. Global agreement and $\epsilon$-agreement are defined as whether the maximum state difference converges to zero and whether the convergence is exponentially

This work has been supported in part by the Knut and Alice Wallenberg Foundation and the Swedish Research Council. G. Shi and K. H. Johansson are with ACCESS Linnaeus Centre, School of Electrical Engineering, Royal Institute of Technology, Stockholm 10044, Sweden. Email: guodongs@kth.se, kallej@ee.kth.se fast, respectively. For the discrete-time case, a necessary and sufficient condition is obtained on $\epsilon$-agreement under general stochasticity, self-confidence, and arc balance assumptions. Then for the continuous-time case, two necessary and sufficient conditions are established on global agreement and $\epsilon$ agreement, respectively. In this way, we precisely state how the persistent graph plays a fundamental role in consensus seeking. Additionally, comparisons of our new conditions are given with existing results and the relations between the discrete-time and continuous-time evolutions are highlighted.

The rest of the paper is organized as follows. In Section II, we introduce the network model and define the problem of interest. Then in Sections III and IV, the main results and convergence analysis are presented for discrete-time and continuous-time dynamics, respectively. Finally concluding remarks are given in Sections V.

\section{Problem Definition}

In this section, we present the network model and define the considered problem. To this end, we first introduce some basic graph theory [4].

A (simple) digraph $\mathcal{G}=(\mathcal{V}, \mathcal{E})$ consists of a finite set $\mathcal{V}=$ $\{1, \ldots, n\}$ of nodes and an arc set $\mathcal{E}$, where each $\operatorname{arc}(i, j) \in$ $\mathcal{E}$ is an ordered pair from node $i \in \mathcal{V}$ to another node $j \in \mathcal{V}$. If the arcs are pairwise distinct in an alternating sequence $v_{0} e_{1} v_{1} e_{2} v_{2} \ldots e_{k} v_{k}$ of nodes $v_{i}$ and $\operatorname{arcs} e_{i}=\left(v_{i-1}, v_{i}\right) \in \mathcal{E}$ for $i=1,2, \ldots, k$, the sequence is called a (directed) path with length $k$. A path from $i$ to $j$ is denoted $i \rightarrow j$, and the length of $i \rightarrow j$ is denoted $|i \rightarrow j|$. A path with no repeated nodes is called a simple path. If there exists a path from node $i$ to node $j$, then node $j$ is said to be reachable from node $i$. Each node is thought to be reachable by itself. A node $v$ from which any other node is reachable is called a center (or a root) of $\mathcal{G} . \mathcal{G}$ is said to be strongly connected if it contains path $i \rightarrow j$ and $j \rightarrow i$ for every pair of nodes $i$ and $j ; \mathcal{G}$ is said to be quasi-strongly connected if $\mathcal{G}$ has a center [5], [25].

The distance from $i$ to $j, d(i, j)$, is defined as the length of a shortest (simple) path $i \rightarrow j$ when $j$ is reachable from $i$, and the diameter of $\mathcal{G}$ as $d_{0}=\max \{d(i, j) \mid i, j \in$ $\mathcal{V}, j$ is reachable from $i$ \}.

In this paper, we consider a network model with node set $\mathcal{V}=\{1, \ldots, n\}$. Let the digraph $\mathcal{G}_{*}=\left(\mathcal{V}, \mathcal{E}_{*}\right)$ denote the underlying graph. The underlying graph indicates all potential interactions between nodes. Node $j$ is said to be a neighbor of $i$ at time $t$ when there is an $\operatorname{arc}(j, i) \in \mathcal{E}_{*}$; each node is supposed to be a neighbor of itself. Let $\mathcal{N}_{i}=$ $\{i\} \cup\left\{j:(j, i) \in \mathcal{E}_{*}\right\}$ denote the neighbor set of node $i$. 


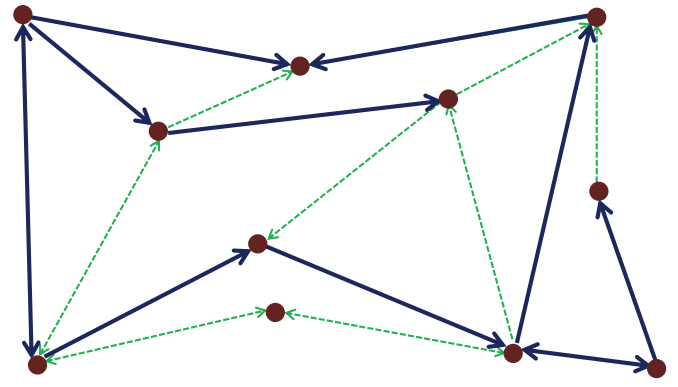

Fig. 1. The underlying graph consists of persistent arcs (solid) and vanishing arcs (dashed). The persistent graph is shown to play a fundamental role for the convergence to an agreement.

Let $x_{i}(t) \in \mathbb{R}$ be the state of node $i$ at time $t$. Time is either discrete or continuous. The initial time is $t_{0} \geq 0$ in both cases and each node is equipped with an initial value $x_{i}\left(t_{0}\right)$. The consensus algorithm is in discrete time:

$$
x_{i}(t+1)=\sum_{j \in \mathcal{N}_{i}} W_{i j}(t) x_{j}(t), \quad i=1, \ldots, n
$$

and in continuous time:

$$
\dot{x}_{i}(t)=\sum_{j \in \mathcal{N}_{i}} W_{i j}(t)\left[x_{j}(t)-x_{i}(t)\right], \quad i=1, \ldots, n .
$$

Here $W_{i j}(t):[0, \infty) \rightarrow[0, \infty)$ is a nonnegative scalar function which represents the weight of arc $(j, i)$. Clearly $W_{i j}(t)$ describes the strength of the influence of node $j$ on $i$. Since $W_{i j}(t)=0$ may happen from time to time, the graph is indeed time-varying.

We define

$$
\psi(t) \doteq \min _{i \in \mathcal{V}}\left\{x_{i}(t)\right\}, \quad \Psi(t) \doteq \max _{i \in \mathcal{V}}\left\{x_{i}(t)\right\}
$$

as the minimum and maximum state value at time $t$, respectively. Then we introduce

$$
\mathcal{H}(t) \doteq \Psi(t)-\psi(t)
$$

The considered global agreement and $\epsilon$-agreement for both the discrete-time and continuous-time updating rules are defined as follows.

Definition 2.1: (a) Global agreement is achieved if for any $x\left(t_{0}\right) \doteq\left(x_{1}\left(t_{0}\right) \ldots x_{n}\left(t_{0}\right)\right)^{T} \in \mathbb{R}^{n}$, we have

$$
\lim _{t \rightarrow \infty} \mathcal{H}(t)=0 \text {. }
$$

(b) Global $\epsilon$-agreement is achieved if there exist two constants $0<\epsilon<1$ and $T_{0}>0$ such that for any $x\left(t_{0}\right) \in \mathbb{R}^{n}$ and $t \geq t_{0}$, we have

$$
\mathcal{H}\left(t+T_{0}\right) \leq \epsilon \mathcal{H}(t) .
$$

The goal of this paper is to distinguish the arcs from the underlying graph that are persistent over a long time range and how they influence global agreement. To be precise, we impose the following definition for persistent arcs and persistent graphs based on the $\mathscr{L}_{1}$ or $\ell_{1}$ norms of the weight functions (see Fig. 1).
Definition 2.2: (a) An $\operatorname{arc}(j, i) \in \mathcal{G}_{*}$ is a persistent arc of the discrete-time updating rule (1) if

$$
\sum_{t=0}^{\infty} W_{i j}(t)=\infty
$$

and a persistent arc of the continuous-time updating rule (2) if

$$
\int_{s}^{\infty} W_{i j}(t) d t=\infty \text { for all } s \geq 0 .
$$

(b) The graph $\mathcal{G}^{p}=\left(\mathcal{V}, \mathcal{E}^{p}\right)$ that consists of all persistent arcs is called the persistent graph.

Next, in Sections III and IV, we will investigate the discrete-time and continuous-time updating rules, respectively. We will establish sufficient and necessary conditions on global agreement and $\epsilon$-agreement, which illustrate that the notion of persistent graphs is critical to the convergence.

\section{DisCRETE-TIME Systems}

In this section, we focus on the discrete-time model (1). In order to obtain the main result, we need the following assumptions.

A1 (Stochasticity) $\sum_{j \in \mathcal{N}_{i}} W_{i j}(t)=1$ for all $i \in \mathcal{V}$ and $t \geq 0$.

A2 (Self-confidence) There exists $0<\eta<1$ such that $W_{i i}(t) \geq \eta$ for $i \in \mathcal{V}$ and $t \geq 0$.

A3 (Arc Balance) There exists a constant $A>1$ such that for any two $\operatorname{arcs}(j, i),(m, k) \in \mathcal{E}^{p}$ and $t \geq 0$, we have

$$
A^{-1} W_{i j}(t) \leq W_{k m}(t) \leq A W_{i j}(t) .
$$

The main result for the discrete-time updating rule (1) on global $\epsilon$-agreement is as follows.

Theorem 3.1: Suppose A1, A2 and A3 hold. Global $\epsilon$ agreement is achieved for (1) if and only if

(a) $\mathcal{G}^{p}$ is quasi-strongly connected;

(b) there exist a constant $a_{*}>0$ and an integer $T_{*}>0$ such that $\sum_{s=t}^{t+T_{*}-1} W_{i j}(s) \geq a_{*}$ for all $t \geq 0$ and $(j, i) \in$ $\mathcal{E}^{p}$.

In fact, if (a) and (b) hold, then we have

$$
\mathcal{H}\left(t+d_{0} T_{*}\right) \leq\left(1-\frac{\eta^{d_{0} T_{*}}}{2} \cdot\left(\frac{a_{*}}{T_{*}}\right)^{d_{0}}\right) \mathcal{H}(t)
$$

for all $t \geq t_{0}$, where $d_{0}$ represents the diameter of $\mathcal{G}^{p}$.

Before we state the proof, we introduce some more notations, which will be used throughout the rest of the paper. For two sets $S_{1}$ and $S_{2}, S_{1} \backslash S_{2}$ is defined as $S_{1} \backslash S_{2}=\{z$ : $\left.z \in S_{1}, z \notin S_{2}\right\}$. For the underlying graph $\mathcal{G}_{*}=\left(\mathcal{V}, \mathcal{E}_{*}\right)$ and the persistent graph $\mathcal{G}^{p}=\left(\mathcal{V}, \mathcal{E}^{p}\right)$, we denote

$$
\begin{gathered}
\theta(t)=\sum_{(j, i) \in \mathcal{E}_{*} \backslash \mathcal{E}^{p}} W_{i j}(t), \\
\xi^{+}(t ; m)=\sum_{j \in \mathcal{N}_{m} \backslash\{m\}} W_{m j}(t),
\end{gathered}
$$

and

$$
\xi_{0}^{+}(t ; m)=\sum_{j \in \mathcal{N}_{m} \backslash\{m\},(j, m) \in \mathcal{E}^{p}} W_{m j}(t) .
$$

In the following two subsections, we prove the necessity and sufficiency parts of Theorem 3.1, respectively. 


\section{A. Necessity}

We need to show that a global $\epsilon$-agreement cannot be achieved without either condition $(a)$ or $(b)$.

The upcoming analysis relies on the following well-known lemmas.

Lemma 3.1: Suppose $0 \leq p_{k}<1$ for all $k$. Then $\sum_{k=0}^{\infty} p_{k}=\infty$ if and only if $\prod_{k=0}^{\infty}\left(1-p_{k}\right)=0$.

Lemma 3.2: $\log (1-t) \geq-2 t$ for all $0 \leq t \leq 1 / 2$.

We have the following proposition indicating that $\mathcal{G}^{p}$ being quasi-strongly connected is not only a necessary condition for (1) to reach global $\epsilon$-agreement, but also necessary for (simple) global agreement, even in the absence of assumptions $\mathrm{A} 2$ and $\mathrm{A} 3$.

Proposition 3.1: Suppose A1 holds. If global agreement is achieved for (1), then $\mathcal{G}^{p}$ is quasi-strongly connected.

We are now in a place to present the following conclusion, which shows the necessity of condition $(b)$ in Theorem 3.1.

Proposition 3.2: Suppose A1 and A3 hold. If global $\epsilon$ agreement is achieved for (1), then there exist a constant $a_{*}>0$ and an integer $T_{*}>0$ such that $\sum_{s=t}^{t+T_{*}} W_{i j}(s) \geq a_{*}$ for all $t \geq 0$ and $(j, i) \in \mathcal{G}^{p}$.

The necessity claim in Theorem 3.1 follows from Propositions 3.1 and 3.2. We refer to [45] for technical details of the proofs.

\section{B. Sufficiency}

We establish a lemma on the upper and lower bounds for some particular nodes.

Lemma 3.3: Suppose A1 holds. Let $x_{m}(t)=\mu \psi(t)+(1-$ $\mu) \Psi(t)$ with $0 \leq \mu \leq 1$. Then for any integer $T>0$, we have:

$$
\begin{aligned}
x_{m}(t+T) \leq & \mu \prod_{s=t}^{t+T-1}\left(1-\xi^{+}(s ; m)\right) \cdot \psi(t) \\
& +\left(1-\mu \prod_{s=t}^{t+T-1}\left(1-\xi^{+}(s ; m)\right)\right) \cdot \Psi(t),
\end{aligned}
$$

and

$$
\begin{aligned}
x_{m}(t+T) \geq & \mu \prod_{s=t}^{t+T-1}\left(1-\xi^{+}(s ; m)\right) \cdot \Psi(t) \\
& +\left(1-\mu \prod_{s=t}^{t+T-1}\left(1-\xi^{+}(s ; m)\right)\right) \cdot \psi(t) .
\end{aligned}
$$

Proof: When $x_{m}(t)=\mu \psi(t)+(1-\mu) \Psi(t)$, for time $t+1$, we have

$$
\begin{aligned}
x_{m}(t+1) \leq & \mu\left(1-\xi^{+}(t ; m)\right) \cdot \psi(t) \\
& +\left(1-\mu\left(1-\xi^{+}(t ; m)\right)\right) \Psi(t) .
\end{aligned}
$$

For time $t+2$, we obtain

$$
\begin{aligned}
x_{m}(t+2) \leq & \mu \prod_{s=t}^{t+1}\left(1-\xi^{+}(s ; m)\right) \cdot \psi(t) \\
& +\left(1-\mu \prod_{s=t}^{t+1}\left(1-\xi^{+}(s ; m)\right)\right) \cdot \Psi(t) .
\end{aligned}
$$

Continuing, we obtain (9).

In equality (10) can be easily obtained using a symmetric analysis as for (9).

We now present the sufficiency proof of Theorem 3.1. In fact, we are going to prove a stronger statement which does not rely on the arc balance assumption A3.

Proposition 3.3: Suppose A1 and A2 hold. Global $\epsilon-$ agreement is achieved for (1) if $\mathcal{G}^{p}$ is quasi-strongly connected and there exist a constant $a_{*}>0$ and an integer $T_{*}>0$ such that $\sum_{s=t}^{t+T_{*}-1} W_{i j}(s) \geq a_{*}$ for all $t \geq 0$ and $(j, i) \in \mathcal{G}^{p}$.

Proof: Let $i_{0} \in \mathcal{V}$ be a center of $\mathcal{G}^{p}$. Take $t_{0} \geq 0$. Assume first that

$$
x_{i_{0}}\left(t_{0}\right) \leq \frac{1}{2} \psi\left(t_{0}\right)+\frac{1}{2} \Psi\left(t_{0}\right) .
$$

Then from Lemma 3.3, one has

$$
\begin{aligned}
x_{i_{0}}\left(t_{0}+T\right) \leq & \frac{1}{2} \prod_{s=t_{0}}^{t_{0}+T-1}\left(1-\xi^{+}\left(s ; i_{0}\right)\right) \cdot \psi\left(t_{0}\right) \\
& +\left(1-\frac{1}{2} \prod_{s=t_{0}}^{t_{0}+T-1}\left(1-\xi^{+}\left(s ; i_{0}\right)\right)\right) \cdot \Psi\left(t_{0}\right) \\
& \leq \frac{\eta^{T}}{2} \psi\left(t_{0}\right)+\left(1-\frac{\eta^{T}}{2}\right) \Psi\left(t_{0}\right)
\end{aligned}
$$

for all $T=0,1, \ldots$.

Denote $\mathcal{V}_{1}$ as the node set consisting of all the nodes of which $i_{0}$ is a neighbor in $\mathcal{G}^{p}$, i.e., $\mathcal{V}_{1}=\left\{j:\left(i_{0}, j\right) \in \mathcal{E}^{p}\right\}$. Note that $\mathcal{V}_{1}$ is nonempty because $i_{0}$ is a center. For any $i_{1} \in \mathcal{V}_{1}$, there exists an instance $\bar{t}_{1} \in\left[t_{0}, t_{0}+T_{*}-1\right]$ such that $W_{i_{1} i_{0}}\left(\bar{t}_{1}\right) \geq a_{*} / T_{*}$ because $\sum_{t=t_{0}}^{t_{0}+T_{*}-1} W_{i_{1} i_{0}}(t) \geq a_{*}$. Suppose $\bar{t}_{1}=t_{0}+\varrho_{1}$ with $\varrho_{1} \in\left[0, T_{*}-1\right]$. Then with (14), we have

$$
\begin{aligned}
x_{i_{1}}\left(\bar{t}_{1}+1\right)= & x_{i_{1}}\left(t_{0}+\varrho_{1}+1\right) \\
\leq & W_{i_{1} i_{0}}\left(t_{0}+\varrho_{1}\right) x_{i_{0}}\left(t_{0}+\varrho_{1}\right) \\
& +\left(1-W_{i_{1} i_{0}}\left(t_{0}+\varrho_{1}\right)\right) \Psi\left(t_{0}\right) \\
\leq & \eta^{\varrho_{1}} \cdot \frac{a_{*}}{2 T_{*}} \cdot \psi\left(t_{0}\right)+\left(1-\eta^{\varrho_{1}} \cdot \frac{a_{*}}{2 T_{*}}\right) \Psi\left(t_{0}\right) .
\end{aligned}
$$

Based on Lemma 3.3, we can further conclude

$$
\begin{aligned}
x_{i_{1}}\left(t_{0}+\varrho_{1}+T\right) \leq & \eta^{\varrho_{1}+T-1} \cdot \frac{a_{*}}{2 T_{*}} \cdot \psi\left(t_{0}\right) \\
& +\left(1-\eta^{\varrho_{1}+T-1} \cdot \frac{a_{*}}{2 T_{*}}\right) \Psi\left(t_{0}\right)
\end{aligned}
$$

for all $T=1,2, \ldots$, which implies

$$
\begin{aligned}
x_{i_{1}}\left(t_{0}+T_{*}+K\right) \leq & \eta^{T_{*}+K} \cdot \frac{a_{*}}{2 T_{*}} \cdot \psi\left(t_{0}\right) \\
& +\left(1-\eta^{T_{*}+K} \cdot \frac{a_{*}}{2 T_{*}}\right) \Psi\left(t_{0}\right)
\end{aligned}
$$

for all $K=0,1, \ldots$.

Next, since $\mathcal{G}^{p}$ is quasi-strongly connected, we can denote $\mathcal{V}_{2}$ as the node set consisting of all the nodes each of which has a neighbor in $\left\{i_{0}\right\} \cup \mathcal{V}_{1}$ within $\mathcal{G}^{p}$. For any $i_{2} \in \mathcal{V}_{2}$, there exist a node $i_{*} \in\left\{i_{0}\right\} \cup \mathcal{V}_{1}$ and an instance $\bar{t}_{2}=t_{0}+T_{*}+\varrho_{2}$ 
with $\varrho_{2} \in\left[0, T_{*}-1\right]$ such that $W_{i_{2} i_{*}}\left(\bar{t}_{1}\right) \geq a_{*} / T_{*}$. Similarly we have

$$
\begin{aligned}
x_{i_{2}}\left(\bar{t}_{2}+1\right) \frac{\eta^{T_{*}+\varrho_{2}}}{2} \cdot\left(\frac{a_{*}}{T_{*}}\right)^{2} \cdot \psi\left(t_{0}\right) & \\
& +\left(1-\frac{\eta^{T_{*}+\varrho_{2}}}{2} \cdot\left(\frac{a_{*}}{T_{*}}\right)^{2}\right) \Psi\left(t_{0}\right),
\end{aligned}
$$

and therefore

$$
\begin{aligned}
x_{i_{2}}\left(t_{0}+2 T_{*}+K\right) \leq & \frac{\eta^{2 T_{*}+K}}{2} \cdot\left(\frac{a_{*}}{T_{*}}\right)^{2} \psi\left(t_{0}\right) \\
& +\left(1-\frac{\eta^{2 T_{*}+K}}{2} \cdot\left(\frac{a_{*}}{T_{*}}\right)^{2}\right) \Psi\left(t_{0}\right)
\end{aligned}
$$

for all $K=0,1, \ldots$.

Proceeding the estimate, $\mathcal{V}_{3}, \ldots, \mathcal{V}_{k}$ can be similarly defined until $\left(\cup_{i=1}^{k} \mathcal{V}_{i}\right) \cup\left\{i_{0}\right\}=\mathcal{V}$. Moreover, it is not hard to see that $i_{0}$ can be selected so that $k=d_{0}$, where $d_{0}$ is the diameter of $\mathcal{G}^{p}$, and thus

$$
\begin{aligned}
\Psi\left(t_{0}+d_{0} T_{*}\right) \leq & \frac{\eta^{d_{0} T_{*}}}{2} \cdot\left(\frac{a_{*}}{T_{*}}\right)^{d_{0}} \cdot \psi\left(t_{0}\right) \\
& +\left(1-\frac{\eta^{d_{0} T_{*}}}{2} \cdot\left(\frac{a_{*}}{T_{*}}\right)^{d_{0}}\right) \Psi\left(t_{0}\right) .
\end{aligned}
$$

With (19), we eventually have

$$
\mathcal{H}\left(t_{0}+d_{0} T_{*}\right) \leq\left(1-\frac{\eta^{d_{0} T_{*}}}{2} \cdot\left(\frac{a_{*}}{T_{*}}\right)^{d_{0}}\right) \mathcal{H}\left(t_{0}\right) .
$$

For the opposite case of (13) with

$$
x_{i_{0}}\left(t_{0}\right)>\frac{1}{2} \psi\left(t_{0}\right)+\frac{1}{2} \Psi\left(t_{0}\right),
$$

(20) is obtained using a symmetric argument by bounding $\psi\left(t_{0}+d_{0} T_{*}\right)$ from below.

Therefore, the desired conclusion follows with $\epsilon=1-$ $\frac{\eta^{d_{0} T_{*}}}{2} \cdot\left(\frac{a_{*}}{T_{*}}\right)^{2}$ and $T_{0}=d_{0} T_{*}$ since (20) holds independent with the choice of $t_{0}$.

\section{Continuous-time Systems}

In this section, we turn to the continuous-time updating rule. We need an assumption on the continuity of each weight function $W_{i j}(t)$ for the existence of trajectories of (2).

A4 (Continuity) Each $W_{i j}(t),(j, i) \in \mathcal{E}_{*}$ is continuous except for a set with measure zero.

With assumption A4, each solution of (2) is considered in the sense of Caratheodory in the following [3], [9].

The upper Dini derivative of a function $h:(a, b) \rightarrow \mathbb{R}$ at $t$ is defined as

$$
D^{+} h(t)=\limsup _{s \rightarrow 0^{+}} \frac{h(t+s)-h(t)}{s}
$$

The next result is useful for the calculation of Dini derivatives [6], [25].

Lemma 4.1: Let $V_{i}(t, x): \mathbb{R} \times \mathbb{R}^{m} \rightarrow \mathbb{R}, i=1, \ldots, n$, be $C^{1}$ and $V(t, x)=\max _{i=1, \ldots, n} V_{i}(t, x)$. If $\mathcal{I}(t)=\{i \in$ $\left.\{1, \ldots, n\}: V(t, x(t))=V_{i}(t, x(t))\right\}$ is the set of indices where the maximum is reached at $t$, then $D^{+} V(t, x(t))=$ $\max _{i \in \mathcal{I}(t)} \dot{V}_{i}(t, x(t))$.
The following lemma establishes the monotonicity of $\Psi(t)$ and $\psi(t)$.

Lemma 4.2: For all $t \geq t_{0} \geq 0$, we have $D^{+} \Psi(t) \leq 0$ and $D^{+} \psi(t) \geq 0$.

Proof: We prove $D^{+} \Psi(t) \leq 0$. The other part can be proved similarly.

Let $\mathcal{I}_{0}(t)$ represent the set containing all the agents that reach the maximum in the definition of $\Psi(t)$ at time $t$, i.e., $\mathcal{I}(t)=\left\{i \in \mathcal{V} \mid x_{i}(t)=\Psi(t)\right\}$. Then according to Lemma 4.1, we obtain

$$
\begin{aligned}
D^{+} \Psi(t) & =\max _{i \in \mathcal{I}_{0}(t)} \dot{x}_{i}(t) \\
& =\max _{i \in \mathcal{I}_{0}(t)}\left[\sum_{j \in N_{i}} W_{i j}(t)\left(x_{j}(t)-x_{i}(t)\right)\right] \\
& \leq 0,
\end{aligned}
$$

which completes the proof.

Lemma 4.2 implies, $\mathcal{H}(t)$ is non-increasing for all $t \geq$ $t_{0}$, and therefore each (Caratheodory) trajectory of (2) is bounded within the initial states of the nodes. As a result, the trajectories exist in $\left[t_{0}, \infty\right)$ for any initial condition.

The main result on global consensus and $\epsilon$-consensus is stated in the following two theorems.

Theorem 4.1: Suppose A3 and A4 hold. Global agreement is achieved for (2) if and only if $\mathcal{G}^{p}$ is quasi-strongly connected.

Theorem 4.2: Suppose A3 and A4 hold. Global $\epsilon$ agreement is achieved for (2) if and only if

(a) $\mathcal{G}^{p}$ is quasi-strongly connected;

(b) there exists two constants $a_{*}, \tau_{0}>0$ such that $\int_{t}^{t+\tau_{0}} W_{i j}(s) d s \geq a_{*}$ for all $t \geq 0$ and $(j, i) \in \mathcal{G}^{p}$.

Moreover, if (a) and (b) hold, then we have

$$
\mathcal{H}\left(t+\tau_{0} \cdot\left\lceil\frac{d_{0} \log 2}{a_{*}}\right\rceil\right) \leq\left(1-\frac{m_{0}^{d_{0}}}{2}\right) \mathcal{H}(t),
$$

where $m_{0}=\left(\frac{\omega_{0}}{2}\right)^{2} \frac{1}{(n-1) A}$ with $\omega_{0}=e^{-\int_{0}^{\infty} \theta(t) d t}, d_{0}$ is the diameter of $\mathcal{G}^{p}$, and $\lceil z\rceil$ represents the smallest integer which is no smaller than $z$.

Theorem 4.1 implies that the connectivity of the persistent graph $\mathcal{G}^{p}$ totally determines whether an agreement can be achieved globally. Furthermore, Theorem 4.2 implies that $\int_{0}^{T} W_{i j}(t) d t=O(T)$ is a critical condition to ensure a global $\epsilon$-consensus.

Remark 4.1: If we have $\int_{t=t_{0}}^{T} W_{i j}(t) d t=\infty,(j, i) \in \mathcal{G}^{p}$ for some finite $T$, it follows from the proof of Theorem 4.1 below that (2) will reach a global agreement in finite time when $t$ tends to $T$.

\section{A. Preliminaries}

We establish two lemmas which describe the boundaries of how much each individual arc affects the nodes' dynamics. We refer to [45] for the technical proofs.

Lemma 4.3: Suppose $x_{m}(s) \leq \mu \psi(s)+(1-\mu) \Psi(s)$ for some $s \geq t_{0}$ and $m \in \mathcal{V}$ with $0 \leq \mu \leq 1$ a giving constant. Then we have

$x_{m}(t) \leq \mu e^{-\int_{s}^{t} \xi^{+}(\tau ; m) d \tau} \psi(s)+\left[1-\mu e^{-\int_{s}^{t} \xi^{+}(\tau ; m) d \tau}\right] \Psi(s)$ 
for all $t \geq s$.

Lemma 4.4: Suppose $(l, m) \in \mathcal{E}_{*}$ and there exists a constant $0<\mu<1$ such that

$$
x_{l}(t) \leq \mu \psi\left(s_{0}\right)+(1-\mu) \Psi\left(s_{0}\right), \quad t \in\left[s_{0}, s\right]
$$

for $t_{0} \leq s_{0}<s$. Then for all $t \in\left[s_{0}, s\right]$, we have

$$
\begin{aligned}
x_{m}(t) \leq & \mu \int_{s_{0}}^{t} e^{-\int_{u}^{t} \xi^{+}(\tau ; m) d \tau} W_{m l}(u) d u \cdot \psi\left(s_{0}\right) \\
& +\left[1-\mu \int_{s_{0}}^{t} e^{-\int_{u}^{t} \xi^{+}(\tau ; m) d \tau} W_{m l}(u) d u\right] \Psi\left(s_{0}\right) .
\end{aligned}
$$

\section{B. Proof of Theorem 4.1}

Let $i_{0} \in \mathcal{V}$ be a center of $\mathcal{G}^{p}$. Assume first that

$$
x_{i_{0}}\left(t_{0}\right) \leq \frac{1}{2} \psi\left(t_{0}\right)+\frac{1}{2} \Psi\left(t_{0}\right)
$$

Denote $\omega_{0}=e^{-\int_{0}^{\infty} \theta(t) d t}$. Then we have $0<\omega_{0} \leq 1$. Thus, based on Lemma 4.3 and noting the fact that $\psi\left(t_{0}\right) \leq \Psi\left(t_{0}\right)$, we have

$$
\begin{aligned}
x_{i_{0}}(t) \leq & \frac{\omega_{0}}{2} e^{-\int_{t_{0}}^{t} \xi_{0}^{+}\left(\tau ; i_{0}\right) d \tau} \psi\left(t_{0}\right) \\
& +\left[1-\frac{\omega_{0}}{2} e^{-\int_{t_{0}}^{t} \xi_{0}^{+}\left(\tau ; i_{0}\right) d \tau}\right] \Psi\left(t_{0}\right) .
\end{aligned}
$$

Define

$$
\hat{t}_{1}=\inf \left\{t \geq t_{0}: e^{-\int_{t_{0}}^{t} \xi_{0}^{+}\left(\tau ; i_{0}\right) d \tau}=\frac{1}{2}\right\} .
$$

We see that $\hat{t}_{1}$ is finite from the definition of $\mathcal{E}^{p}$. As a result, we obtain

$$
x_{i_{0}}(t) \leq \frac{\omega_{0}}{4} \psi\left(t_{0}\right)+\left[1-\frac{\omega_{0}}{4}\right] \Psi\left(t_{0}\right), \quad t \in\left[t_{0}, \hat{t}_{1}\right] .
$$

Next, we denote the node set consisting of all the nodes of which $i_{0}$ is a neighbor in $\mathcal{G}^{p}$ as $\mathcal{V}_{1}$, i.e., $\mathcal{V}_{1}=\left\{j:\left(i_{0}, j\right) \in\right.$ $\left.\mathcal{E}^{p}\right\}$. Note that $\mathcal{V}_{1}$ is nonempty because $i_{0}$ is a center. Then for any $i_{1} \in \mathcal{V}_{1}$, we see from Lemma 4.4 that

$$
\begin{aligned}
x_{i_{1}}\left(\hat{t}_{1}\right) \leq & \frac{\omega_{0}^{2}}{4} \int_{t_{0}}^{\hat{t}_{1}} e^{-\int_{u}^{\hat{t}_{1}} \xi_{0}^{+}\left(\tau ; i_{1}\right) d \tau} W_{i_{1} i_{0}}(u) d u \cdot \psi\left(t_{0}\right) \\
& +\left[1-\frac{\omega_{0}^{2}}{4} \int_{t_{0}}^{\hat{t}_{1}} e^{-\int_{u}^{\hat{t}_{1}} \xi_{0}^{+}\left(\tau ; i_{1}\right) d \tau} W_{i_{1} i_{0}}(u) d u\right] \Psi\left(s_{0}\right) .
\end{aligned}
$$

The arc balance assumption A3 implies that

$$
\int_{u}^{\hat{t}_{1}} \xi_{0}^{+}\left(t ; i_{1}\right) d t \leq \int_{u}^{\hat{t}_{1}}(n-1) A W_{i_{1} i_{0}}(t) d t
$$

which yields

$$
\begin{aligned}
& \int_{t_{0}}^{\hat{t}_{1}} e^{-\int_{u}^{\hat{t}_{1}} \xi_{0}^{+}\left(\tau ; i_{1}\right) d \tau} W_{i_{1} i_{0}}(u) d u \\
\geq & \frac{1}{(n-1) A} \cdot\left[1-e^{-(n-1) A \int_{t_{0}}^{\hat{t}_{1}} W_{i_{1} i_{0}}(\tau) d \tau}\right] .
\end{aligned}
$$

On the other hand, we also have

$$
\int_{t_{0}}^{\hat{t}_{1}} \xi_{0}^{+}\left(t ; i_{0}\right) d t \leq \int_{t_{0}}^{\hat{t}_{1}}(n-1) A W_{i_{1} i_{0}}(t) d t .
$$

Thus, we know from (29) and the definition of $\hat{t}_{1}$ that

$$
\begin{aligned}
& \int_{t_{0}}^{\hat{t}_{1}} e^{-\int_{u}^{\hat{t}_{1}} \xi_{0}^{+}\left(\tau ; i_{1}\right) d \tau} W_{i_{1} i_{0}}(u) d u \\
\geq & \frac{1}{(n-1) A} \cdot\left[1-e^{-\int_{t_{0}}^{\hat{t}_{1}} \xi_{0}^{+}\left(\tau ; i_{0}\right) d \tau}\right] \\
= & \frac{1}{2(n-1) A} .
\end{aligned}
$$

Equations (28) and (30) result in

$$
x_{i_{1}}\left(\hat{t}_{1}\right) \leq \frac{m_{0}}{2} \psi\left(t_{0}\right)+\left(1-\frac{m_{0}}{2}\right) \Psi\left(t_{0}\right)
$$

for all $i_{1} \in \mathcal{V}_{1}$, where $m_{0}=\left(\frac{\omega_{0}}{2}\right)^{2} \frac{1}{(n-1) A}$.

Since $\mathcal{G}^{p}$ has a center, we can proceed the estimation to nodes in $\mathcal{V}_{2}, \ldots, \mathcal{V}_{k}$ until $\left(\cup_{j=1}^{k} \mathcal{V}_{j}\right) \cup\left\{i_{0}\right\}=\mathcal{V}$ with $\hat{t}_{2}, \ldots, \hat{t}_{k}$ such that

$$
x_{i}\left(\hat{t}_{k}\right) \leq \frac{m_{0}^{k}}{2} \psi\left(t_{0}\right)+\left(1-\frac{m_{0}^{k}}{2}\right) \Psi\left(t_{0}\right)
$$

for all $i \in \mathcal{V}$, which leads to

$$
\Psi\left(\hat{t}_{k}\right) \leq \frac{m_{0}^{k}}{2} \psi\left(t_{0}\right)+\left(1-\frac{m_{0}^{k}}{2}\right) \Psi\left(t_{0}\right) .
$$

We see that $i_{0}$ can be chosen so that $k \leq d_{0}$ always holds, where $d_{0}$ is the diameter of $\mathcal{G}^{p}$. Denoting $t_{1}=\hat{t}_{k}$, we eventually arrive at

$$
\begin{aligned}
\mathcal{H}\left(t_{1}\right) & \leq \frac{m_{0}^{d_{0}}}{2} \psi\left(t_{0}\right)+\left(1-\frac{m_{0}^{d_{0}}}{2}\right) \Psi\left(t_{0}\right)-\psi\left(t_{0}\right) \\
& =\left(1-\frac{m_{0}^{d_{0}}}{2}\right) \mathcal{H}\left(t_{0}\right)
\end{aligned}
$$

Although the analysis up to now is based on assumption (25), we see that (34) also holds for the other case with $x_{i_{0}}\left(t_{0}\right)>\frac{1}{2} \psi\left(t_{0}\right)+\frac{1}{2} \Psi\left(t_{0}\right)$ using a symmetric argument by investigating the lower bound of $\psi\left(t_{1}\right)$.

Similar estimate can be carried out for $t_{k}, k=2,3, \ldots$, which leads to

$$
\mathcal{H}\left(t_{k+1}\right) \leq\left(1-\frac{m_{0}^{d_{0}}}{2}\right) \mathcal{H}\left(t_{k}\right)
$$

for all $t_{k}, k=1,2, \ldots$, which yields

$$
\mathcal{H}\left(t_{k}\right) \leq\left(1-\frac{m_{0}^{d_{0}}}{2}\right)^{k} \mathcal{H}\left(t_{0}\right) .
$$

Therefore, we can now conclude that $\lim _{t \rightarrow \infty} \mathcal{H}(t)=0$ because $\mathcal{H}(t)$ is non-increasing and $0<m_{0}<1$. The sufficiency statement of Theorem 4.1 is thus proved.

The necessity part follows the same line as the proof of Proposition 3.1, and therefore omitted.

\section{Proof of Theorem 4.2}

The necessity statement follows from a similar argument as the proof of Proposition 3.2. The sufficiency part can be obtained based on the convergence analysis in Theorem 4.1. We refer to [45] for technical details. 


\section{COnClusions}

This paper studied persistent graphs under discrete-time and continuous-time consensus algorithms. Sufficient and necessary conditions were established on the persistent graph for the network to reach global agreement or $\epsilon$-agreement. It was shown that the persistent graph essentially determines both the convergence and convergence rate to an agreement.

\section{REFERENCES}

[1] D. P. Bertsekas and J. N. Tsitsiklis. Parallel and Distributed Computation: Numerical Methods, Prentice Hall, 1989.

[2] F. Clarke, Y. Ledyaev, R. Stern, and P. Wolenski. Nonsmooth Analysis and Control Theory. Speringer-Verlag, 1998.

[3] A. F. Filippov. Differential Equations with Discontinuous Righthand Sides. Norwell, MA: Kluwer, 1988.

[4] C. Godsil and G. Royle. Algebraic Graph Theory. New York: SpringerVerlag, 2001.

[5] C. Berge and A. Ghouila-Houri. Programming, Games, and Transportation Networks. John Wiley and Sons, New York, 1965.

[6] J. Danskin. The theory of max-min, with applications. SIAM J. Appl. Math., vol. 14, 641-664, 1966.

[7] J. Wolfowitz, "Products of indecomposable, aperiodic, stochastic matrices," Proc. Amer. Math. Soc., vol. 15, pp. 733-736, 1963.

[8] J. Hajnal, "Weak Ergodicity in Non-homogeneous Markov Chains," Proc. Cambridge Philos. Soc., no. 54, pp. 233-246, 1958.

[9] J. Cortés, "Discontinuous dynamical systems-a tutorial on solutions, nonsmooth analysis, and stability," IEEE Control Systems Magazine, vol. 28 , no. 3, 36-73, 2008.

[10] M. H. DeGroot, "Reaching a consensus," Journal of the American Statistical Association, vol. 69, no. 345, pp. 118-121, 1974.

[11] P. M. DeMarzo, D. Vayanos, J. Zwiebel, "Persuasion bias, social influence, and unidimensional opinions," Quarterly Journal of Economics, vol. 118 , no. 3 , pp. $909-968,2003$

[12] B. Golub and M. O. Jackson, "Naïve learning in social networks and the wisdom of crowds," American Economic Journal: Microeconomics, vol. 2, no. 1, pp. 112-149, 2007.

[13] D. Acemoglu, A. Ozdaglar and A. ParandehGheibi, "Spread of (Mis)information in Social Networks," Games and Economic Behavior, vol. 70, no. 2, pp. 194-227, 2010.

[14] D. Acemoglu, G. Como, F. Fagnani, and A. Ozdaglar, "Opinion fluctuations and persistent disagreement in social networks," 50th IEEE Conference on Decision and Control, Orlando, Florida, December 2011.

[15] J. N. Tsitsiklis, D. Bertsekas, and M. Athans, "Distributed asynchronous deterministic and stochastic gradient optimization algorithms," IEEE Trans. Automatic Control, vol. 31, no. 9, pp. 803-812, 1986.

[16] A. Jadbabaie, J. Lin, and A. S. Morse, "Coordination of groups of mobile autonomous agents using nearest neighbor rules," IEEE Trans. Automatic Control, vol. 48, no. 6, pp. 988-1001, 2003.

[17] R. Olfati-Saber and R. Murray, "Consensus problems in the networks of agents with switching topology and time dealys," IEEE Trans. Automatic Control, vol. 49, no. 9, pp. 1520-1533, 2004.

[18] S. Boyd, A. Ghosh, B. Prabhakar and D. Shah, "Randomized gossip algorithms," IEEE Trans. Information Theory, vol. 52, no. 6, pp. 2508$2530,2006$.

[19] J. Fax and R. Murray, "Information flow and cooperative control of vehicle formations," IEEE Trans. Automatic Control, vol. 49, no. 9, pp. 1465-1476, 2004.

[20] L. Moreau, "Stability of multiagent systems with time-dependent communication links," IEEE Trans. Automatic Control, vol. 50, no. 2, pp. 169-182, 2005.

[21] W. Ren and R. Beard, "Consensus seeking in multiagent systems under dynamically changing interaction topologies," IEEE Trans. on Automatic Control, vol. 50, no. 5, pp. 655-661, 2005.

[22] S. Martinez, J. Cortés, and F. Bullo, "Motion coordination with distributed information," IEEE Control Systems Magazine, vol. 27, no. 4, pp. 75-88, 2007.

[23] H. G. Tanner, A. Jadbabaie, G. J. Pappas, "Flocking in fixed and switching networks," IEEE Trans. Automatic Control, vol. 52, no. 5, pp. 863-868, 2007.

[24] Y. Hong, L. Gao, D. Cheng, and J. Hu, "Lyapuov-based approach to multi-agent systems with switching jointly connected interconnection," IEEE Trans. Automatic Control, vol. 52, pp. 943-948, 2007.
[25] Z. Lin, B. Francis, and M. Maggiore, "State agreement for continuoustime coupled nonlinear systems," SIAM J. Control Optim., vol. 46, no. 1, pp. 288-307, 2007.

[26] F. Xiao and L. Wang, "Asynchronous consensus in continuous-time multi-agent systems with switching topology and time-varying delays,' IEEE Trans. Automatic Control, vol. 53, no. 8, pp. 1804-1816, 2008.

[27] M. Cao, A. S. Morse and B. D. O. Anderson, "Reaching a consensus in a dynamically changing environment: a graphical approach," SIAM J. Control Optim., vol. 47, no. 2, pp. 575-600, 2008.

[28] M. Cao, A. S. Morse and B. D. O. Anderson, "Reaching a consensus in a dynamically changing environment: convergence rates, measurement delays, and asynchronous events," SIAM J. Control Optim., vol. 47, no. 2, pp. 601-623, 2008.

[29] D. Cheng, J. Wang, and X. Hu, "An extension of LaSalle's invariance principle and its application to multi-agents consensus," IEEE Trans. Automatic Control, vol. 53, pp. 1765-1770, 2008.

[30] G. Shi and Y. Hong, "Global target aggregation and state agreement of nonlinear multi-agent systems with switching topologies," Automatica, vol. 45, pp. 1165-1175, 2009.

[31] G. Shi, Y. Hong and K. H. Johansson, "Connectivity and set tracking of multi-agent systems guided by multiple moving leaders," IEEE Trans. Automatic Control, to appear.

[32] S. Kar and J. M. F. Moura, "Distributed consensus algorithms in sensor networks: link failures and channel noise," IEEE Trans. on Signal Processing, vol. 57, no. 1, pp. 355-369, 2009.

[33] S. Kar and J. M. F. Moura, "Distributed consensus algorithms in sensor networks: quantized data and random link failures, IEEE Trans. Signal Processing, vol. 58, no. 3, pp. 1383-1400, 2010.

[34] S. Patterson, B. Bamieh and A. El Abbadi, "Convergence rates of distributed average consensus with stochastic link failures," IEEE Trans. Autom. Control, vol. 55, no. 4, pp. 880-892, 2010.

[35] F. Fagnani and S. Zampieri, "Randomized consensus algorithms over large scale networks," IEEE J. on Selected Areas of Communications, vol. 26, no. 4, pp. 634-649, 2008.

[36] S. Muthukrishnan, B. Ghosh, and M. Schultz, "First and second order diffusive methods for rapid, coarse, distributed load balancing," Theory of Computing Systems, vol. 31, pp. 331-354, 1998.

[37] R. Diekmann, A. Frommer, and B. Monien, "Efficient schemes for nearest neighbor load balancing," Parallel Computing, vol. 25, pp. 789812,1999

[38] C. Intanagonwiwat, R. Govindan, and D. Estrin, "Directed diffusion: a scalable and robust communication paradigm for sensor networks," in Proceedings ACM/IEEE Conf. Mobile Computing and Networking, pp. $56-67,2000$.

[39] D. Kempe, A. Dobra, and J. Gehrke, "Gossip-based computation of aggregate information," in Proc. Conf. Foundations of Computer Science, pp. 482-491, 2003.

[40] A. Nedić, A. Olshevsky, A. Ozdaglar, and J. N. Tsitsiklis, "On distributed averaging algorithms and quantization effects," IEEE Trans. Automatic Control, vol. 54, no. 11, pp. 2506-2517, 2009.

[41] A. Olshevsky and J. N. Tsitsiklis, "Convergence speed in distributed consensus and averaging," SIAM J. Control Optim., vol. 48, no.1, pp. $33-55,2009$.

[42] A. Olshevsky and J. N. Tsitsiklis, "Degree fluctuations and the convergence time of consensus algorithms," 50th IEEE Conference on Decision and Control, Orlando, Florida, December 2011.

[43] J. M. Hendrickx and J. N. Tsitsiklis, "Convergence of type-symmetric and cut-balanced consensus seeking systems," submitted, available online from arXiv:1102.2361v1.

[44] B. Touri and A. Nedić, "Alternative characterization of ergodicity for doubly stochastic chains," 50th IEEE Conference on Decision and Control, Orlando, Florida, December 2011.

[45] G. Shi and K. H. Johansson, "The role of persistent graphs in the agreement seeking of social networks," preprint at arxiv.org, avaiable from http://arxiv.org/pdf/1112.1338v2.pdf 